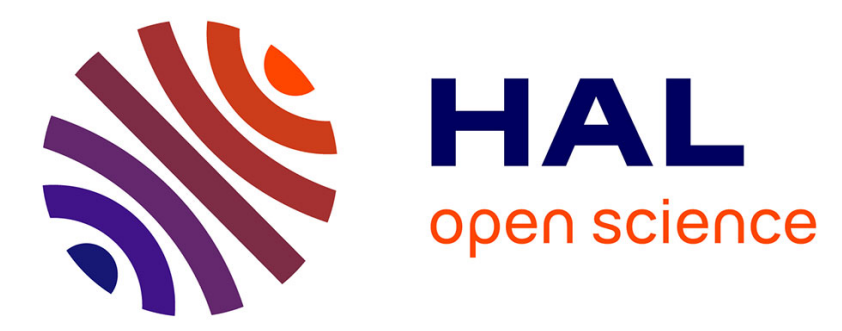

\title{
Bifurcation of inflated circular cylinders of elastic material under axial loading-II. Exact theory for thick-walled tubes
}

\author{
David Haughton, Raymond Ogden
}

\section{To cite this version:}

David Haughton, Raymond Ogden. Bifurcation of inflated circular cylinders of elastic material under axial loading-II. Exact theory for thick-walled tubes. Journal of the Mechanics and Physics of Solids, 1979, 10.1016/0022-5096(79)90027-9 . hal-01302310

\author{
HAL Id: hal-01302310 \\ https://hal.science/hal-01302310
}

Submitted on 14 Apr 2016

HAL is a multi-disciplinary open access archive for the deposit and dissemination of scientific research documents, whether they are published or not. The documents may come from teaching and research institutions in France or abroad, or from public or private research centers.
L'archive ouverte pluridisciplinaire HAL, est destinée au dépôt et à la diffusion de documents scientifiques de niveau recherche, publiés ou non, émanant des établissements d'enseignement et de recherche français ou étrangers, des laboratoires publics ou privés.

\section{다(1)(2)}

Distributed under a Creative Commons Attribution - ShareAlike| 4.0 International 


\title{
BIFURCATION OF INFLATED CIRCULAR CYLINDERS OF ELASTIC MATERIAL UNDER AXIAL LOADING-II. EXACT THEORY FOR THICK-WALLED TUBES
}

\author{
D. M. Haughton and R. W. Ogden
}

School of Mathematics, University of Bath, Claverton Down, Bath BA2 7AY, England

\begin{abstract}
BIFURCATION from a circular cylindrical configuration of an elastic tube of finite wall thickness is examined when the loading is that of an axial force combined with internal or external pressure. This extends the analysis given by D. M. HAUGHTON and R. W. OGDEN (1979), (Part I of this series), for membrane cylinders. The relative importance of prismatic, axisymmetric and asymmetric bifurcations for axial tension and compression combined with internal or external pressure is discussed. In particular, the dependence on wall-thickness, ratio of length to radius, and magnitude of the axial stretch is detailed. Some analytical results are presented for a general form of incompressible isotropic elastic strain-energy function. For the most part, however, it is necessary to solve the equations numerically. This is done for specific forms of the strain-energy function.
\end{abstract}

\section{INTRODUCTION}

In Haughton and Ogden (1979), (Part I of this series, henceforth referred to as (I)), detailed results were given relating to the bifurcation from a circular cylindrical configuration of an elastic membrane tube subjected to combined axial loading and internal pressure. In the present paper, Part II of this series, an investigation of the corresponding problems for tubes of finite wall thickness is carried out. The results in (I) serve as a guide through the more difficult analysis required for the nonhomogeneous deformations arising in thick-walled tubes.

As in (I), prismatic, axisymmetric and asymmetric modes of bifurcation are examined. A limited number of analytical results are found for an arbitrary form of incompressible, isotropic elastic strain-energy function, and specific details then discussed for a particular class of strain-energy functions. Analytical results are not obtainable for most bifurcations even for very simple forms of strain-energy function so the governing differential equations are solved numerically. The numerical results are illustrated for four specific forms of strain-energy function.

For thick-walled circular cylindrical tubes the prismatic type of bifurcation has been examined by several authors. In particular, Hill (1976) has obtained analytical results for the " $n=1$ " bifurcation mode using a method different from that described here. WANG and ERTEPINAR (1972) consider a thick-walled tube subjected to an external pressure and no axial force. They give specific results for the neo-Hookean 
form of strain-energy function, as also do NowINSKI and SHAHINPOOR (1969) who confine attention to infinitely-long tubes. However, for externally-pressurized tubes, bifurcation occurs at "small" strains and the results obtained are essentially independent of the precise form of the strain-energy function. We have confirmed this by calculations carried out for several different forms of strain-energy function. Under external pressure it is found, moreover, that a prismatic mode occurs before any possible axisymmetric mode.

The internally pressurized thick-walled tube, which appears not to have been studied previously, provides a contrast to the above results. When the tube is subjected to an internal pressure combined with axial tension the axisymmetric type of bifurcation is found to dominate and the behaviour exhibited is similar in character to that found in (I) for a membrane.

For a tube subjected to internal pressure and axial compression, results are given which, in the limit of zero pressure, coincide with those obtained by WILKES (1955), who examined the stability of a thick tube subjected to an end-thrust and gave specific results for the neo-Hookean form of strain-energy function. For the same problem, with attention restricted to the neo-Hookean form of strain-energy function from the outset, Sierakowski, SUn and Ebcioglu (1975) have similarly investigated axisymmetric modes of deformation. PATterson (1976), also for the neo-Hookean form of strain-energy function, obtains numerical results for asymmetric deformation modes when axial compression is combined with external pressure.

Results are given here for the combination of internal pressure and axial loading (tensile and compressive). The relative importance of the prismatic, axisymmetric and asymmetric bifurcations is discussed in relation to (i) the thickness of the cylinder wall, (ii) the ratio of the length to radius of the cylinder, and (iii) the magnitude of the axial stretch.

\section{The Finitely-deformed Circular Cylindrical Configuration}

We suppose that the thick-walled cylindrical shell is defined by

$$
A \leqslant R \leqslant B, \quad 0 \leqslant \Theta \leqslant 2 \pi, \quad 0 \leqslant Z \leqslant L
$$

in its undeformed configuration, where $R, \Theta, Z$ are cylindrical polar coordinates.

The cylinder is subjected to an axial loading and an internal pressure so that the circular cylindrical shape is maintained, and the shell defined by

$$
a \leqslant r \leqslant b, \quad 0 \leqslant \theta \leqslant 2 \pi, \quad 0 \leqslant z \leqslant l,
$$

where $r, \theta, z$ are cylindrical polar coordinates in the current (deformed) configuration.

Under the assumption that the material is incompressible, the deformation is described by the equations

$$
r^{2}=\lambda_{z}^{-1}\left(R^{2}-A^{2}\right)+a^{2}, \quad \theta=\Theta, \quad z=\lambda_{z} Z,
$$

where $\lambda_{z}$ is the axial extension ratio.

Let $\mathbf{e}_{1}, \mathbf{e}_{2}, \mathbf{e}_{3}$ denote the unit basis vectors corresponding to the coordinates $\theta, z, r$ respectively and let $\lambda_{1}, \lambda_{2}, \lambda_{3}$ be the corresponding principal stretches. 
From the incompressibility constraint we have

$$
\lambda_{1} \lambda_{2} \lambda_{3}=1
$$

while from (3),

$$
\lambda_{2}=\lambda_{z}, \quad \lambda_{1}=\frac{r}{R} \equiv \lambda, \quad \lambda_{3}=\left(\lambda \lambda_{z}\right)^{-1}
$$

where we have used (4) and introduced the azimuthal stretch $\lambda$.

We regard $\lambda_{z}$ as a given constant and $\lambda$ as a function of $r$ (or $R$ ) from (3).

For an incompressible isotropic elastic solid the strain-energy function $W \equiv W\left(\lambda_{1}, \lambda_{2}, \lambda_{3}\right)$, per unit volume, depends symmetrically on $\lambda_{1}, \lambda_{2}, \lambda_{3}$. We denote the principal components of the Cauchy stress tensor $\sigma$ by $\sigma_{i i}(i=1,2,3)$, $\dagger$ so that

where

$$
\sigma_{i i}=\sigma_{i}-p \quad(i=1,2,3)
$$

$$
\sigma_{i}=\lambda_{i} \frac{\partial W}{\partial \lambda_{i}} \quad(i=1,2,3),
$$

and $p$ is the arbitrary hydrostatic pressure arising from the incompressibility constraint.

Use of (5)-(7) leads to

where

$$
\sigma_{11}-\sigma_{33}=\lambda \hat{W}_{\lambda}, \quad \sigma_{22}-\sigma_{33}=\lambda_{z} \hat{W}_{\lambda_{z}}
$$

$$
\hat{W}\left(\lambda, \lambda_{z}\right)=W\left(\lambda, \lambda_{z},\left(\lambda \lambda_{z}\right)^{-1}\right)=\hat{W}\left(\lambda_{z}, \lambda\right),
$$

and $\hat{W}_{\lambda}, \hat{W}_{\lambda_{z}}$ denote $\partial \hat{W} / \partial \lambda, \partial \hat{W} / \partial \lambda_{z}$.

Equations (8) are similar in form to those given in (I) for a membrane except that $\sigma_{33} \neq 0$ here and there is now dependence on the radius $r$.

For the symmetric configuration considered here, the only equilibrium equation not satisfied trivially is

$$
r \frac{d \sigma_{33}}{d r}+\sigma_{33}-\sigma_{11}=0,
$$

it being assumed that there are no body forces.

If $\sigma_{33}$ is $-P$ on $r=a$ and zero on $r=b$, where $P$ denotes the internal pressure, integration of (10) leads to

$$
P=\int_{a}^{b} \lambda \hat{W}_{\lambda} \frac{d r}{r} \equiv \int_{\lambda_{b}}^{\lambda_{a}} \frac{\hat{W}_{\lambda} d \lambda}{\left(\lambda^{2} \lambda_{z}-1\right)} .
$$

In deriving (11) use has been made of $\left(8_{1}\right)$ and we have adopted the notation $\lambda_{a}=a / A, \lambda_{b}=b / B$, which, from $\left(3_{1}\right)$, gives

$$
\lambda_{a}^{2} \lambda_{z}-1=\frac{R^{2}}{A^{2}}\left(\lambda^{2} \lambda_{z}-1\right)=\eta\left(\lambda_{b}^{2} \lambda_{z}-1\right)
$$

where

$$
\eta=B^{2} / A^{2} \geqslant 1
$$

$\dagger$ The subscript $i$ is not summed here or in the sequel. 
Note that equality in (13) corresponds to the membrane limit discussed in (I), while from (12) it follows that

$$
\lambda_{a} \geqslant \lambda \geqslant \lambda_{b}
$$

with equality holding if and only if $P=0$. (In this connection we recall from (I) that $\hat{W}_{\lambda} \gtrless 0$ according as $\hat{\lambda}^{2} \lambda_{z} \gtrless 1$.)

The change of independent variable from $r$ to $\lambda$ in $(11)$ is achieved by means of $\left(3_{1}\right)$ and the definition $\left(5_{2}\right)$.

In view of (12) we regard $P$ as a function of $\lambda_{z}$ and $\lambda_{a}$. Differentiation of (11) with respect to $\lambda_{a}$ then gives

$$
\lambda_{a}^{-1}\left(\lambda_{a}^{2} \lambda_{z}-1\right) \frac{\partial P}{\partial \lambda_{a}}=\hat{W}_{\lambda}\left(\lambda_{a}, \lambda_{z}\right) / \lambda_{a}-\hat{W}_{\lambda}\left(\lambda_{b}, \lambda_{z}\right) / \hat{\lambda}_{b}
$$

This is a straightforward generalization of a formula given by OGDEN (1978, equation (36)) for $\lambda_{z}=1$.

Equation (15) should be compared with the corresponding equation for a membrane given in (I, equation (36)) to which it reduces for "small" $(B-A) / A$.

Clearly, a necessary condition for pressure turning-points to exist at fixed $\hat{\lambda}_{z}$ is that

$$
\lambda^{-1} \hat{W}_{\lambda} \text { is not monotonic in } \lambda \text {, }
$$

and for (16) to hold it is necessary that

$$
\lambda \hat{W}_{\lambda \lambda}-\hat{W}_{\lambda}=0
$$

for some $\lambda$ in the interval $\left(\lambda_{b}, \lambda_{a}\right)$.

As shown in (I), the equation (17) characterizes the pressure turning-points in the case of a membrane. Analogous considerations in respect of a spherical shell have been discussed by HAUGHTON and OGDEN (1978). Experimental data indicating the occurrence of a pressure maximum for thick-walled tubes have been obtained by Skala (1970) and Charrier and Li (1977).

The resultant axial loading $N$ on the ends of the cylinder is given by

$$
N=2 \pi \int_{a}^{b} \sigma_{22} r d r .
$$

Use of (8) and (10) enables this to be expressed as

$$
N / \pi A^{2}=\left(\lambda_{a}^{2} \lambda_{z}-1\right) \int_{\lambda_{b}}^{\lambda_{a}} \frac{\left(2 \lambda_{z} \hat{W}_{\lambda_{z}}-\lambda \hat{W}_{\lambda}\right) \lambda d \lambda}{\left(\lambda^{2} \lambda_{z}-1\right)^{2}}+P \lambda_{a}^{2} .
$$

The corresponding expression for a membrane is $2 \varepsilon \hat{W}_{\lambda_{z}}$ where $\varepsilon=H / R, H$ being the thickness of the membrane and $R$ its radius in the undeformed configuration. In (I) it was shown to be reasonable for the inequalities $\hat{W}_{\lambda_{z} \lambda_{z}}>0, W_{\lambda i_{z}}>0$ to hold, where $\lambda$ is the azimuthal stretch in the membrane. It is also reasonable for these inequalities to hold for the thick-walled specimen, but in general it cannot be expected that the analogous global inequalities $\partial N / \partial \lambda_{z}>0, \partial N / \partial \lambda_{a}>0$ are valid in view of the complicated form of $N$. 
Since $\sigma_{33} \neq 0$, in general, for a thick-walled cylinder, the local form of the axial stress obtained from $\left(8_{2}\right)$ yields

$$
\left.\begin{array}{l}
F\left(\lambda_{a}, \lambda_{z}\right)=\hat{W}_{\lambda_{z}}\left(\lambda_{a}, \lambda_{z}\right)-\lambda_{z}^{-1} P, \\
F\left(\lambda_{b}, \lambda_{z}\right)=\hat{W}_{\lambda_{z}}\left(\lambda_{b}, \lambda_{z}\right),
\end{array}\right\}
$$

where $F\left(\lambda, \lambda_{z}\right)=\lambda_{z}^{-1} \sigma_{22}$ is the axial stress per unit undeformed area of cross-section (nominal stress). Even for these local forms it cannot in general be deduced that $\partial F\left(\lambda_{a}, \lambda_{z}\right) / \partial \lambda_{z}>0$ or $\partial F\left(\lambda_{a}, \lambda_{z}\right) / \partial \lambda_{a}>0$. Moreover, $\partial F\left(\lambda_{b}, \lambda_{z}\right) / \partial \lambda_{z}>0$ at fixed $\lambda_{a}$ is not a consequence of $\hat{W}_{\lambda_{z} \lambda_{z}}>0$ since $\lambda_{b}$ depends on $\lambda_{z}$ through (12).

Since $N$ may be treated as a function of $\lambda_{a}$ and $\lambda_{z}$, one consequence of the above discussion is that inflation is not necessarily accompanied by axial shortening of the tube at fixed $N$. That is,

$$
\frac{d \lambda_{z}}{d \lambda_{a}} \equiv-\frac{\partial N}{\partial \lambda_{a}} / \frac{\partial N}{\partial \lambda_{z}}
$$

may be positive for certain values of $\lambda_{a}$ and $\lambda_{z}$, in contrast to the situation for a membrane (see (I)). Indeed, CHADWICK and HADDON (1972) have provided an example to illustrate that a tube can extend during inflation in the absence of axial loading.

If $\sigma_{22}^{\prime}$ denotes the deviatoric part of the axial stress $\sigma_{22}$ and $N^{\prime}$ its resultant, then it is easily shown, directly from (8), that

$$
N^{\prime} / \pi A^{2}=\frac{2}{3}\left(\lambda_{a}^{2} \lambda_{z}-1\right) \int_{\lambda_{b}}^{\lambda_{a}} \frac{2 \lambda_{z} \hat{W}_{\lambda_{z}}-\lambda \hat{W}_{\lambda}}{\left(\lambda^{2} \hat{\lambda}_{z}-1\right)^{2}} \lambda d \lambda .
$$

The significance of this expression lies in the fact that for a tube with closed ends (so there is a contribution $P \pi a^{2}$ to the axial stress) (18) is replaced by $N=3 N^{\prime} / 2$. We shall return to this point later in relation to the discussion of numerical results (Section 4).

\section{The InCREmental Equations}

Full details of the notation and equations used here are given in HAUGHTON and OGDEN (1978). Here we summarize briefly the relevant information.

The incremental equilibrium equations (in the absence of body forces) can be written

$$
\operatorname{div} \dot{\mathbf{s}}_{0}=0 \text {, }
$$

where $\dot{\mathbf{s}}_{0}$ is the increment in nominal stress referred to the current configuration (indicated by the subscript zero).

The incremental boundary conditions in respect of a hydrostatic pressure loading are

$$
\dot{\mathbf{s}}_{0}^{\mathrm{T}} \mathbf{n}=P \mathbf{\eta}^{\mathrm{T}} \mathbf{n}-\dot{P} \mathbf{n}
$$

evaluated on the appropriate boundary, where superscript $T$ denotes the transpose, $n$ 
is the unit normal in the current configuration and $\boldsymbol{\eta}=\dot{\boldsymbol{\alpha}}_{0}, \dot{\boldsymbol{\alpha}}$ being the increment in the deformation gradient $\boldsymbol{\alpha}$.

The incremental constitutive law is written

$$
\dot{\mathbf{s}}_{0}=\mathscr{B} \boldsymbol{\eta}+p \boldsymbol{\eta}-\dot{p} \boldsymbol{\delta},
$$

where $\mathscr{B}$ is the fourth-order tensor of instantaneous moduli associated with the conjugate variables $(\mathbf{s}, \boldsymbol{\alpha})$ and $\boldsymbol{\delta}$ denotes the identity tensor.

The components of $\mathscr{B}$ on the principal axes of the underlying deformation are given by Haughton and Ogden (1978, equations (84)-(86)) and therefore not repeated here.

With the basis vectors defined in Section 2 and the choice $\theta, z, r$ of coordinates, (20) becomes

$$
\dot{S}_{o j i, j}+\dot{s}_{o j i} \mathbf{e}_{k} \cdot \mathbf{e}_{j, k}+\dot{s}_{o k j} \mathbf{e}_{i} \cdot \mathbf{e}_{j, k}=0 \quad(i=1,2,3),
$$

where the non-zero values of $\mathbf{e}_{i} \cdot \mathbf{e}_{j, k}$ are precisely those given for $\mathbf{a}_{i} \cdot \mathbf{a}_{j, k}$ in (I, equation (23)). In the present situation, however, $r$ varies through the thickness of the shell.

We write the increment $\dot{\mathbf{x}}$ in the position vector $\mathbf{x}$ of a point in the current configuration as

$$
\dot{\mathbf{x}}=v \mathbf{e}_{1}+w \mathbf{e}_{2}+u \mathbf{e}_{3} .
$$

Then, the components of $\boldsymbol{\eta}$ on the basis $\mathbf{e}_{1}, \mathbf{e}_{2}, \mathbf{e}_{3}$ are displayed as

$$
\left[\begin{array}{ccc}
\left(u+v_{\theta}\right) / r & v_{z} & v_{r} \\
w_{\theta} / r & w_{z} & w_{r} \\
\left(u_{\theta}-v\right) / r & u_{z} & u_{r}
\end{array}\right],
$$

where the subscripts $\theta, z, r$ denote partial derivatives.

Since the material is incompressible, we have

$$
\operatorname{tr}(\boldsymbol{\eta}) \equiv u_{r}+\left(u+v_{\theta}\right) / r+w_{z}=0
$$

\section{Bifurcation Analysis}

\subsection{Prismatic bifurcations}

Firstly, we assume that $u, v, w$ are independent of $z$. Then (22)-(26) together with the expressions for the components $: \mathscr{B}_{i j k l}$ from Haughton and OGDEN (1978) give

$$
\begin{aligned}
& \dot{p}_{r}=\left[r\left(\mathscr{B}_{3333}^{\prime}-\mathscr{B}_{1133}^{\prime}+p^{\prime}\right)+\mathscr{B}_{3333}+\mathscr{B}_{1111}-2 \mathscr{B}_{1133}\right] u_{r} / r+\left(\mathscr{B}_{3333}-\mathscr{B}_{1133}\right) u_{r r} \\
& +\mathscr{B}_{1313}\left(u_{\theta \theta}-v_{\theta}\right) / r^{2}+\mathscr{B}_{1331} v_{r \theta} / r \text {. } \\
& \dot{p}_{\theta}=\left[r\left(\cdot \mathscr{B}_{1331}^{\prime}+p^{\prime}\right)+\mathscr{B}_{1313}\right]\left(u_{\theta}-v\right) / r+\left(r: \mathscr{B}_{3131}^{\prime}+\mathscr{B}_{3131}\right) v_{r} \\
& +\not B_{3131} r v_{r r}+\left(. B_{1331}+\mathscr{B}_{1133}-. B_{1111}\right) u_{r \theta}, \\
& \mathscr{B}_{1212} w_{\theta \theta}^{\prime} / r+\mathscr{B}_{3232}^{\prime} r w_{r}^{\prime}+\mathscr{B}_{3232}\left(r w_{r r}+w_{r}\right)=0 \text {, }
\end{aligned}
$$

where the prime denotes $d / d r$. 
Equation (29) can be solved quite generally for $w$ at any stage of the deformation and, in particular, in the undeformed configuration. The solution has no effect on the shape of the cylinder cross-section and deserves no further consideration in the present context. We therefore set $w=0$, as in the membrane case treated in (I).

The boundary conditions (21) on the lateral surfaces of the tube become

$$
u_{\theta}-v+r v_{r}=0 \text { on } r=a, b
$$

and

$$
\left(B_{3333}+\sigma_{3}-B_{1133}\right) u_{r}-\dot{p}=\left\{\begin{array}{ll}
-\dot{P} & \text { on } r=a, \\
0 & \text { on } r=b .
\end{array}\right\}
$$

In the derivation of $(30)$ it has been assumed that $\mathscr{B}_{3131} \neq 0$. In fact, the inequality $: B_{3131}>0$, a consequence of the Baker-Ericksen inequalities, is adopted here.

To solve equations (27) and (28) with the incompressibility condition (26) we set

$$
\left.\begin{array}{l}
u=f_{n}(r) \cos n \theta, \\
v=g_{n}(r) \sin n \theta, \\
\dot{p}=h_{n}(r) \cos n \theta,
\end{array}\right\}
$$

for example. (In general, four independent functions of $\theta$ are associated with each function of $r$ on the right side of (32).)

Equation (26) gives

$$
r f_{n}^{\prime}+f_{n}+n g_{n}=0, \quad n \geqslant 0 .
$$

This is used to eliminate $h_{n}$ and $g_{n}$ from the other equations, leading to

$$
\begin{aligned}
& r\left\{B_{3131} r^{3} f_{n}^{\prime \prime \prime}+\left(r: B_{3131}^{\prime}+3 \cdot B_{3131}\right) r^{2} f_{n}^{\prime \prime}\right. \\
& \left.+\left[r: B_{3131}^{\prime}-\mathscr{B}_{3131}+n^{2}\left(2 \mathscr{B}_{1331}+2 \mathscr{B}_{1133}-\mathscr{B}_{1111}-\mathscr{B}_{3333}\right)\right] r f_{n}^{\prime}\right\}^{\prime} \\
& \quad+\left(n^{2}-1\right)\left[r^{2} \cdot \mathscr{B}_{3131}^{\prime \prime}+r \cdot \mathscr{B}_{3131}^{\prime}+\left(n^{2}-1\right) \mathscr{B}_{3131}+n^{2}\left(\sigma_{1}-\sigma_{3}\right)\right] f_{n}=0
\end{aligned}
$$

for $n \geqslant 1$.

Correspondingly, the boundary conditions (30) and (31) for $n \geqslant 1$ yield

$$
r^{2} f_{n}^{\prime \prime}+r f_{n}^{\prime}+\left(n^{2}-1\right) f_{n}=0 \quad \text { on } r=a, b
$$

and, after some rearrangements,

$$
\begin{aligned}
& \mathscr{B}_{3131} r^{3} f_{n}^{\prime \prime \prime}+\left(r . B_{3131}^{\prime}+4 \mathscr{B}_{3131}\right) r^{2} f_{n}^{\prime \prime}+ \\
& +\left[r \cdot \mathscr{B}_{3131}^{\prime}-\left(n^{2}-1\right) \cdot B_{3131}+n^{2}\left(2 \cdot B_{1331}+2 \mathscr{B}_{1133}-\mathscr{B}_{1111}-\mathscr{B}_{3333}\right)\right] r f_{n}^{\prime} \\
& +\left(n^{2}-1\right)\left(r \cdot \not_{3131}^{\prime}+\mathscr{B}_{3131}\right) f_{n}=0 \text { on } r=a, b .
\end{aligned}
$$

For $n=0,(31)$ gives

$$
\left(\not B_{3333}-\not B_{1133}+\sigma_{3}\right) f_{0} / r+h_{0}=\left\{\begin{array}{ll}
\dot{P} & \text { on } r=a, \\
0 & \text { on } r=b,
\end{array}\right\}
$$

where $h_{0}$ is obtained from

$$
r^{2} h_{0}^{\prime}=\left(r \cdot \mathscr{B}_{1133}^{\prime}-r \cdot \mathscr{B}_{3333}^{\prime}-r p^{\prime}+\mathscr{B}_{3333}-\mathscr{B}_{1111}\right) f_{0},
$$

and (30) is satisfied identically. 
Equations equivalent to (34), (35) and (36) were given by WANG and ErTePINAR (1972), amongst others, for the special case of a neo-Hookean solid, and by HILL (1976) for a general form of strain-energy function.

For $n=0$, the circular symmetry is maintained and the analysis is equivalent to that described in Section 2. No further discussion of this is necessary.

For $n=1$, equation (34) is integrated to give

$$
\begin{aligned}
. \mathscr{B}_{3131} r^{3} f_{1}^{\prime \prime \prime}+ & \left(r \cdot \mathscr{B}_{3131}^{\prime}+3 \cdot \mathscr{B}_{3131}\right) r^{2} f_{1}^{\prime \prime} \\
& +\left(r \cdot \mathscr{B}_{3131}^{\prime}-\cdot \mathscr{B}_{3131}+2 \cdot \mathscr{B}_{1331}+2 \cdot \mathscr{B}_{1133}-\mathscr{B}_{1111}-\mathscr{B}_{3333}\right) r f_{1}^{\prime}=0 .
\end{aligned}
$$

The constant of integration has been set equal to zero to ensure that the boundary conditions (35) and (36) are compatible. Use of (40) in (36) for $n=1$ shows that each of $(35)$ and $(36)$ reduces to

$$
r f_{1}^{\prime \prime}+f_{1}^{\prime}=0 \quad \text { on } r=a, b .
$$

Use of the formula $r \lambda^{\prime}=\lambda\left(1-\lambda^{2} \lambda_{z}\right)$ enables two further integrations to be carried out. These, consecutively, give

and

$$
r f_{1}^{\prime \prime}+\left(2+\lambda^{2} \lambda_{z}\right) f_{1}^{\prime}=c_{1} r / \lambda_{1 / B_{3131}},
$$

$$
r^{3} f_{1}^{\prime} / \lambda=c_{1} \int \frac{r^{3} d r}{\lambda^{2}-B_{3131}}+c_{2},
$$

where $c_{1}$ and $c_{2}$ are constants.

Since the differential equations and boundary conditions are homogeneous, $f_{1}=$ const. is a solution. Thus, for prismatic bifurcations to occur, a non-zero $f_{1}^{\prime}$ is required. Substitution of (42) into (40) leads to the bifurcation criterion

$$
\int_{\lambda_{b}}^{\lambda_{a}} \frac{\hat{W}_{\lambda \lambda} \lambda d \lambda}{\left(\lambda^{2} \lambda_{z}-1\right)\left(\hat{W}_{\lambda}\right)^{2}}=0,
$$

use having been made of the connection

$$
\mathscr{B}_{3131}=\lambda \hat{W}_{\lambda} /\left(\lambda^{4} \lambda_{z}^{2}-1\right) .
$$

In view of (12), equation (43) determines the bifurcation points $\lambda_{a}$ (dependent on $\lambda_{z}$ ). It is applicable equally to internally or externally pressurized cylinders at fixed axial extension.

The special case $P=0$ corresponds to $\lambda^{2} \lambda_{z}=1$, that is $\hat{W}_{\lambda} \equiv 0$. Separate analysis of this case directly from the differential equations and boundary conditions (noting that the underlying deformation is now homogeneous) shows that prismatic bifurcation is not possible for any mode number $n$ except in the limit $b \rightarrow a$.

From (43) a necessary condition for $n=1$ mode prismatic bifurcation to occur is $\hat{W}_{\lambda \lambda}=0$ for some $\lambda$ in $\left(\lambda_{b}, \lambda_{a}\right)$. In the membrane limit, equation (43) reduces to a necessary and sufficient condition, namely $\hat{W}_{\lambda \lambda}=0$ for some $\lambda$ (as shown directly in (I)).

However, arguments presented in (I) suggest that the inequality

$$
\hat{W}_{\lambda \lambda}>0
$$

holds for all $\lambda$ and $\lambda_{z}$, ensuring that prismatic bifurcations are not possible for a 
membrane. For a thick-walled cylinder, the inequality (44) excludes $n=1$ mode prismatic bifurcation but higher-order modes remain possible. We remark that although (44) is physically reasonable for a membrane it could possibly be violated within the bulk of a thick material specimen. Nevertheless, we adopt (44) here.

For mode numbers $n \geqslant 2$, WANG and ERTEPINAR (1972) have considered the problem of an externally pressurized thick-walled tube in respect of the neo-Hookean form of strain-energy function, restricting attention to $\lambda_{z}=1$. Under external pressure, the strains at bifurcation are "small" and the results obtainable essentially independent of the precise form of strain-energy function. We have confirmed this by calculations for a number of different strain-energy functions.

We use the class of strain-energy functions described fully in (I). This is such that

$$
\hat{W}\left(\lambda, \lambda_{z}\right)=\sum_{r} \mu_{r}\left\{\lambda^{\alpha_{r}}+\lambda_{z}^{\alpha_{r}}+\left(\lambda \lambda_{z}\right)^{-\alpha_{r}}-3\right\} / \alpha_{r} .
$$

In particular, in Table 1, we show our calculations in respect of the three-term form of (45) with material constants given by

$$
\begin{aligned}
& \left.\alpha_{1}=1 \cdot 3, \quad \alpha_{2}=5 \cdot 0, \quad \alpha_{3}=-2 \cdot 0, \quad\right\} \\
& \left.\mu_{1}^{*}=1.491, \quad \mu_{2}^{*}=0.003, \quad \mu_{3}^{*}=-0.023,\right\}
\end{aligned}
$$

where $\mu_{r}^{*}=\mu_{r} / \mu$ and $\mu$ is the ground-state shear modulus (see (I)).

In Table 1 , values of $\lambda_{b}$ at bifurcation are given for $\lambda_{z}=1,2,3,4,5$ and 0.5 for a number of different values of the thickness ratio $A / B$ in respect of (45) with (46). For comparison we reproduce the results of ERTEPINAR (1972) for $\lambda_{z}=1$ and the neoHookean form of strain-energy function. In all cases it is found to be the $n=2$ mode which occurs first. We remark that for $A / B=0.95$ the critical values of $\lambda_{b}$ for $\lambda_{z} \geqslant 1$ correspond to $P=0$ to within an accuracy of three decimal places, this being consistent with the situation in the membrane limit.

Under internal pressure our calculations reveal that no prismatic mode of bifurcation is possible for the strain-energy functions considered.

In Fig. 1 the pressure $P$, as given by (11), is plotted as a function of $\lambda_{a}$ in respect of (45) with (46) for a number of values of $\lambda_{z}$ and for $A / B=0 \cdot 85$. Because the value of $P$

\begin{tabular}{|c|c|c|c|c|c|c|c|}
\hline \multirow[b]{2}{*}{$A / B$} & \multirow{2}{*}{$\begin{array}{c}\begin{array}{c}\text { neo-Hookean } \\
\text { (Ertepinar, } \\
1972)\end{array} \\
1\end{array}$} & \multicolumn{6}{|c|}{ Three-term strain-energy function } \\
\hline & & 1 & 2 & 3 & 4 & 5 & 0.5 \\
\hline 0.95 & 0.999 & 0.999 & 0.707 & 0.577 & 0.500 & 0.447 & 1.413 \\
\hline $0 \cdot 9$ & 0.997 & 0.997 & 0.705 & 0.576 & 0.499 & 0.446 & $1 \cdot 410$ \\
\hline $0 \cdot 85$ & 0.994 & 0.994 & 0.703 & 0.574 & 0.497 & 0.445 & 1.406 \\
\hline $0 \cdot 8$ & 0.989 & 0.990 & $0 \cdot 700$ & 0.571 & 0.495 & 0.443 & 1.400 \\
\hline 0.7 & 0.979 & 0.979 & 0.693 & 0.566 & 0.490 & 0.438 & $1 \cdot 385$ \\
\hline $0 \cdot 6$ & 0.965 & 0.968 & 0.685 & 0.559 & $0 \cdot 485$ & 0.434 & $1 \cdot 369$ \\
\hline
\end{tabular}

TABLE 1. Values of $\lambda_{b}$ at bifurcation for various values of $\lambda_{z}$ and $A / B$ in respect of (45) with (46) 


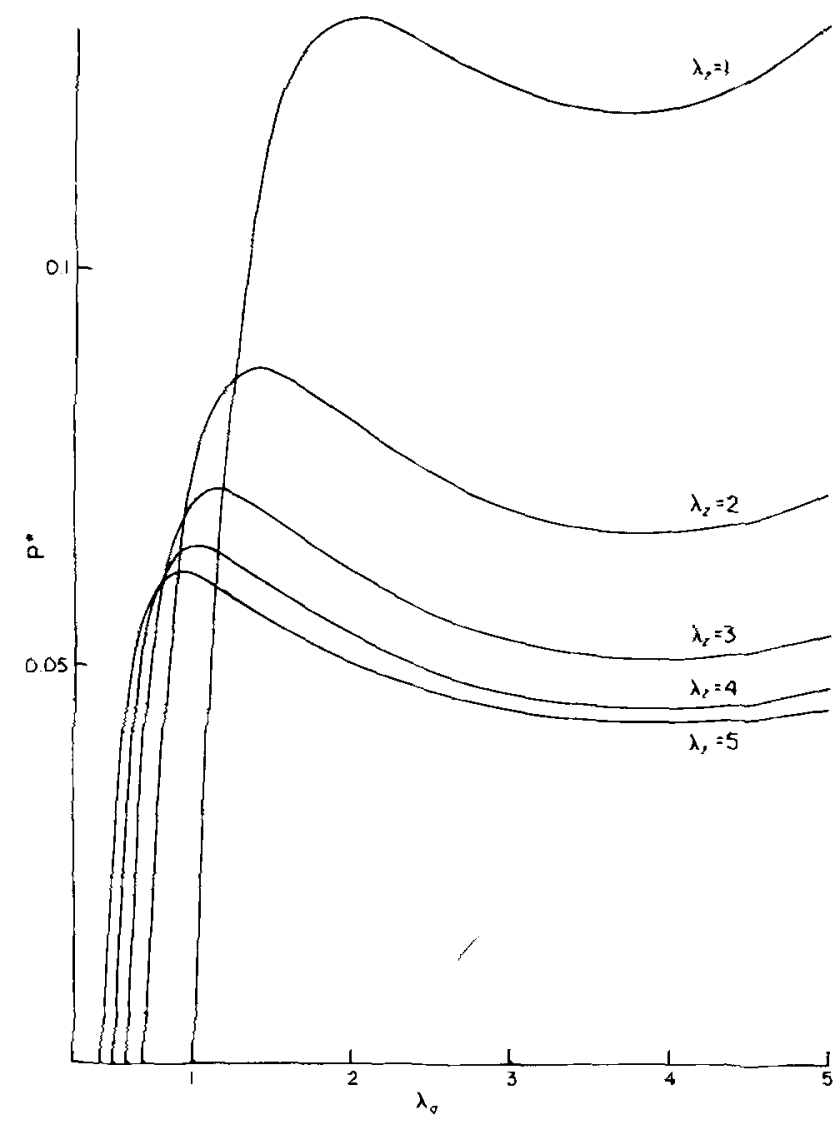

FIG. 1. Plot of the pressure $P^{*}=P / \mu$ against $\lambda_{a}$ for $\lambda_{z}=1,2,3,4,5$ with $A / B=0 \cdot 85$.

increases rapidly with $B / A$ we do not include corresponding curves for smaller values of $A / B$. Instead, in Fig. 2, the value of $\lambda_{a}$ at the pressure turning-points is plotted against $A / B$. For suffeciently large wall thicknesses, pressure turning-points do not occur for all values of $\lambda_{z}$, but this is not shown in Fig. 2.

So far no mention has been made of the boundary conditions on the ends of the cylinder. These can be chosen either to exclude prismatic bifurcations or to be compatible with them. They are more important in the discussion of axisymmetric and asymmetric bifurcations, which we consider next.

\subsection{Axisymmetric bifurcations}

When equations (23) are independent of $\theta$ the equation for $v$ decouples from those for $u$ and $w$. The solutions of this equation are of little interest in the present context and it is considered no further. The remaining equations become

$$
\begin{aligned}
\dot{p}_{r}=\left(r: \mathscr{B}_{1133}^{\prime}-\mathscr{B}_{111}\right) u / r^{2}+\left(r \mathscr{B}_{3333}^{\prime}+r p^{\prime}+\mathscr{B}_{3333}\right) u_{r} / r+\mathscr{B}_{3333} u_{r r} \\
+\mathscr{B}_{2323} u_{z 2}+\left(r \cdot \mathscr{B}_{2233}^{\prime}+\mathscr{B}_{2233}-\mathscr{B}_{1122}\right) w_{z} / r+\left(\mathscr{B}_{2233}+\mathscr{B}_{3223}\right) w_{r z},
\end{aligned}
$$




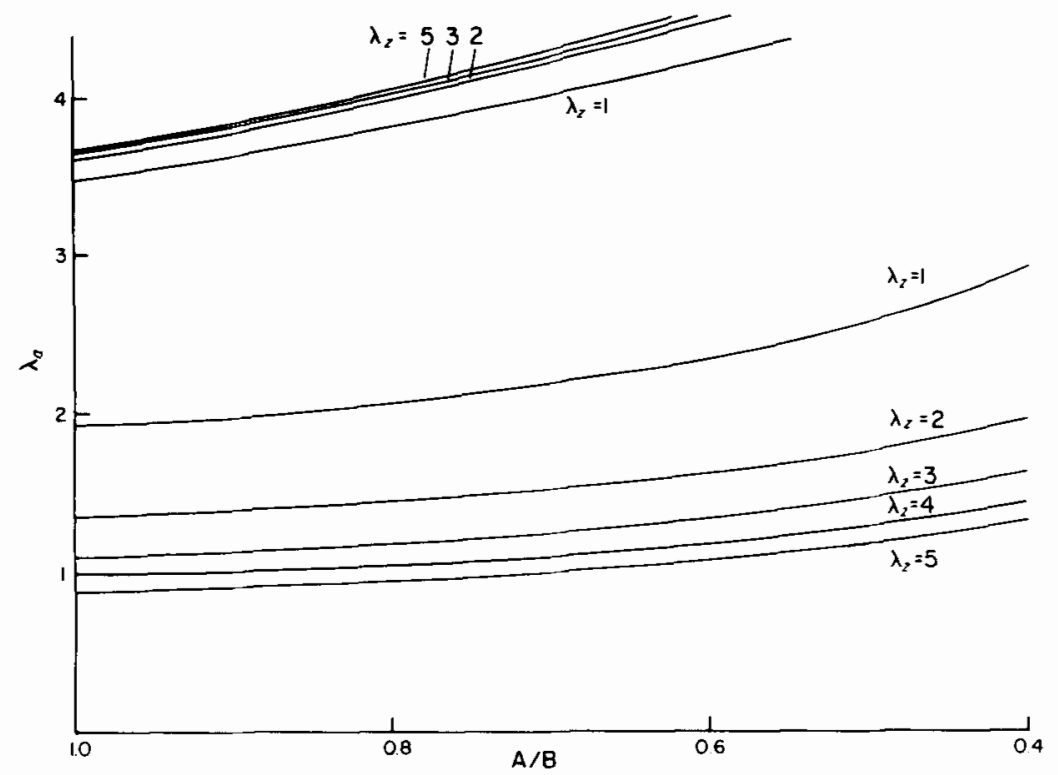

FIG. 2. Plot of the values of $\lambda_{a}$ corresponding to $\partial P / \partial \lambda_{a}=0$ against $A / B$ for $\lambda_{z}=1,2,3,4,5$.

$$
\begin{aligned}
\dot{p}_{z}=\mathscr{B}_{3232} w_{r r}+\left(r \mathscr{B}_{3232}^{\prime}+\mathscr{B}_{3232}\right) w_{r} / r+\mathscr{B}_{2222} w_{z z}+\left(\mathscr{B}_{2233}+\mathscr{B}_{3223}\right) u_{r z} \\
+\left(r \mathscr{B}_{3223}^{\prime}+r p^{\prime}+\mathscr{B}_{3223}+\mathscr{B}_{1122}\right) u_{z} / r,
\end{aligned}
$$

and (26) reduces to

$$
u_{r}+u / r+w_{2}=0 .
$$

The boundary conditions $(21)$ on the lateral surface yield

$$
\left(\mathscr{B}_{3333}-\mathscr{B}_{2233}+\sigma_{3}\right) u_{r}+\left(\mathscr{B}_{1133}-\mathscr{B}_{2233}\right) u / r-\dot{p}=0 \text { on } r=a, b
$$

and

$$
w_{r}+u_{z}=0 \quad \text { on } r=a, b .
$$

The equations and boundary conditions have been made homogeneous by the omission of the incremental pressure $\dot{P}$, this being associated with a circular cylindrical solution as discussed in Section 2.

In order to solve the above system, we write

for example $(\alpha \neq 0)$.

$$
u=f(r) \sin \alpha z
$$

Elimination of $w$ and $\dot{p}$ between (47), (48) and (49) leads to

$$
\begin{aligned}
r^{4}\left[\mathscr{B}_{3232} f^{\prime \prime \prime}+\left(r \mathscr{B}_{3232}^{\prime}+2 \mathscr{B}_{3232}\right) f^{\prime \prime} / r+\left(r \mathscr{B}_{3232}^{\prime}-\mathscr{B}_{3232}\right) f^{\prime} / r^{2}\right. \\
\left.-\left(r \mathscr{B}_{3232}^{\prime}-\mathscr{B}_{3232}\right) f / r^{3}\right]^{\prime}+\alpha^{2} r^{2}\left[\left(2 \mathscr{B}_{2233}+2 \mathscr{B}_{3223}-\mathscr{B}_{3333}-\mathscr{B}_{2222}\right) r^{2} f^{\prime \prime}\right. \\
+\left(2 r \mathscr{B}_{3223}^{\prime}+2 r \mathscr{B}_{2233}^{\prime}-r \mathscr{B}_{3333}^{\prime}-r \mathscr{B}_{2222}^{\prime}-\mathscr{B}_{3333}-\mathscr{B}_{2222}+2 \mathscr{B}_{2233}+2 \mathscr{B}_{3223}\right) r f^{\prime} \\
+\left(r^{2} \mathscr{B}_{3223}^{\prime \prime}+r^{2} p^{\prime \prime}+r \mathscr{B}_{3223}^{\prime}+r \mathscr{B}_{1122}^{\prime}-r \mathscr{B}_{1133}^{\prime}-r \mathscr{B}_{2222}^{\prime}+r \mathscr{B}_{2233}^{\prime}\right. \\
\left.\left.\quad+\mathscr{B}_{1111}+\mathscr{B}_{2222}-2 \mathscr{B}_{1122}-2 \mathscr{B}_{3223}\right) f\right]+\alpha^{4} r^{4} \mathscr{B}_{2323} f=0,
\end{aligned}
$$


while the boundary conditions (50) and (51) can be written

$$
\begin{aligned}
& \mathscr{B}_{3232} r^{3} f^{\prime \prime \prime}+\left(r \cdot \not_{3232}^{\prime}+2 \cdot \not B_{3232}\right) r^{2} f^{\prime \prime}+\left(r \cdot \mathscr{B}_{3232}^{\prime}-\mathscr{B}_{3232}\right) r f^{\prime}-\left(r: \mathscr{B}_{3232}^{\prime}-. \mathscr{B}_{3232}\right) f \\
& -\alpha^{2} r^{2}\left[\left(\mathscr{B}_{3333}+\mathscr{B}_{2222}-2 \mathscr{B}_{2233}-\mathscr{B}_{3223}+\sigma_{3}\right) r f^{\prime}\right. \\
& \left.-\left(r \cdot B_{3232}^{\prime}-r \sigma_{33}^{\prime}+\not B_{3232}-\sigma_{3}+\mathscr{B}_{1122}-\mathscr{B}_{2222}+\mathscr{B}_{2233}-\mathscr{B}_{1133}\right) f\right]=0 \\
& \text { on } r=a, b \text {, } \\
& r^{2} f^{\prime \prime}+r f^{\prime}+\left(\alpha^{2} r^{2}-1\right) f=0 \quad \text { on } r=a, b .
\end{aligned}
$$

To fit the boundary conditions on the ends of the cylinder we write

$$
u=\left\{\begin{array}{ll}
f_{\alpha}(r)(A \sin \alpha z+B \cos \alpha z), & \alpha \neq 0, \\
f_{0}(r)(A z+B) & \alpha=0,
\end{array}\right\}
$$

where $A$ and $B$ are constants. The corresponding form of $w$, obtained from (49) and consistent with (51), is

$$
w=\left\{\begin{array}{ll}
{\left[f_{\alpha}^{\prime}(r)+f_{\alpha}(r) / r\right](A \cos \alpha z-B \sin \alpha z) / \alpha,} & \alpha \neq 0, \\
-\left[f_{0}^{\prime}(r)+f_{0}(r) / r\right]\left(\frac{1}{2} A z^{2}+B z\right)+C_{0}(r), & \alpha=0,
\end{array}\right\}
$$

where $C_{0}(r)$ is a function of $r$ related to $f_{0}(r)$, while $\dot{p}$, if required, can be obtained from (47) and (48).

To be specific we impose the end conditions

$$
\dot{s}_{023}=w=0 \quad \text { on } z=0, l,
$$

which are satisfied by (56) and (57) provided

$$
u=f_{\alpha}(r) \cos \alpha z, \quad w=-\left[f_{\alpha}^{\prime}(r)+f_{\alpha}(r) / r\right](\sin \alpha z) / \alpha,
$$

where

$$
\alpha l=n \pi \quad(n=1,2,3, \ldots) .
$$

Note that the solution corresponding to $\alpha=0$ is ruled out by (58) and we can take $\alpha>0$ without loss of generality. The boundary conditions (58) correspond to zero shear stress and zero axial displacement pointwise on the ends of the tube.

The values of $\alpha$ l given by (60) are also obtained if the end conditions (58) are replaced by ones in which the (nominal) stress is held fixed pointwise and is in the axial direction. If the resultant axial loading on the end faces is held fixed, on the other hand, values of $\alpha l$ depending on $\lambda_{z}$ and $\lambda_{a}$ may be obtained. Such dependence arises in the membrane case, treated in (I), in which integration with respect to $r$ through the thickness of the material is implicit from the outset, and the solution analogous to $\left(56_{1}\right)$ involves two (in general, distinct) values of $\alpha$. Note that (56) is not the most general solution for $u$ since (53) is quadratic in $\alpha^{2}$. For a thick-walled tube the use of "average" end conditions involves lengthy algebra, and we therefore confine attention to pointwise end-conditions which, although yielding simple and explicit values of $\alpha l$, nevertheless allow the main features of the bifurcation phenomena to be revealed. By the same token it suffices to consider only (58) and (60).

Because the length of the cylinder is at our disposal, we can arbitrarily set $n=1$ in (60). The value

$$
\alpha=\pi / l=\pi / \lambda_{z} L
$$


is then substituted in the differential equation (53) in order to obtain $f_{\alpha}(r)$ subject to (54) and (55) for different choices of the dimensionless geometric parameters $L / B$ and $A / B$, recalling (1).

Results calculated on the basis of (61) show how the length of the cylinder affects the onset of axisymmetric bifurcation into the $n=1$ mode. Equally, and equivalently, if $L$ is fixed and $n$ varied then results show how a cylinder of fixed length responds to axisymmetric bifurcations in different mode numbers. This dual interpretation has been exploited in (I) and we continue it here. The spectrum of values of $\alpha$ from 0 to $\infty$ embraces cylinders of arbitrary length for any mode number.

The differential equation (53) cannot in general be solved analytically, even for very simple forms of strain-energy function, except in the case $\alpha=0$ when a simple explicit form for $f_{0}(r)$ can be written down. For finite $L, \alpha=0$ corresponds to maintenance of the circular cylindrical shape and the solution is $u=A / r$ and $w=0$, where $A$ is a constant. In the limit $L \rightarrow \infty$, when $\alpha \rightarrow 0$, the solution (59) reduces to

$$
u=(A / r) \sin \alpha z, \quad w=0,
$$

with $\alpha$ l remaining finite, and these expressions replace (56) and (57) for this case.

The solution of (53) with $\alpha=\pi / \lambda_{z} L$, together with the boundary conditions (54) and (55), leads to a bifurcation criterion which is a relation between $\lambda_{z}$ and $\lambda_{a}$ (or $\lambda_{b}$ in view of (12)). Numerical calculations have been carried out for a number of values of $\lambda_{2}$ and the ratio $L / B$. The numerical method is described in Section 4.3.

In Fig. 3(a,b, c) for $L / B=20,10,5$ respectively, the bifurcation curves corresponding to $A / B=0.85$ are shown in the $\left(\lambda_{z}, \lambda_{a}\right)$-plane. Results for the neoHookean, Mooney-Rivlin and Varga forms of strain-energy function are compared with those for the three-term strain-energy function (46). Corresponding results are given in Fig. $4(\mathrm{a}, \mathrm{b}, \mathrm{c})$ for $A / B=0.5$.

In each case, the curve of $P=0$ is also shown and attention is restricted to $P \geqslant 0$. Regions of both (axial) tension and compression are included. The curves of pressure turning-points (independent of $L / B$ ), calculated by setting $\partial P / \partial \lambda_{a}=0$ in (15), are plotted in Figs 3 and 4 in respect of the three-term strain-energy function (46). These show that, dependent on $\lambda_{z}, A / B$ and $L / B$, bifurcation may occur before or after the pressure maximum during inflation, just as for a membrane (see (I)).

For reasons discussed by Haughton and OGDEN $(1978,1979)$ the neo-Hookean, Mooney-Rivlin and Varga forms of strain-energy function exclude certain bifurcation features. The remainder of our calculations is therefore based on the three-term strain-energy function (46). We note from Figs 3 and 4, however, that there is little difference between predictions of the several strain-energy functions in the region of axial compression $\left(\lambda_{z}<1\right)$.

In respect of (46) the bifurcation curves corresponding to axial compression are shown on a larger scale in Fig. $5(\mathrm{a}, \mathrm{b})$ for $A / B=0.85$ and 0.5 respectively. In each case, the values $L / B=5,10,20$ are chosen. The curve of zero resultant axial force (that is, $N=0$, where $N$ is given by $(18)$ ) is included in Fig. $5(\mathrm{a}, \mathrm{b})$ to emphasize the boundary between the regimes of resultant axial tension and compression.

We note that as the axial compression is increased in the absence of pressure (that is, along the curve $\lambda_{a}^{2} \lambda_{2}=1$ ), the value of $\lambda_{a}$ at bifurcation increases with the value of $L / B$. At first sight this is at variance with one's intuition that long tubes buckle more 


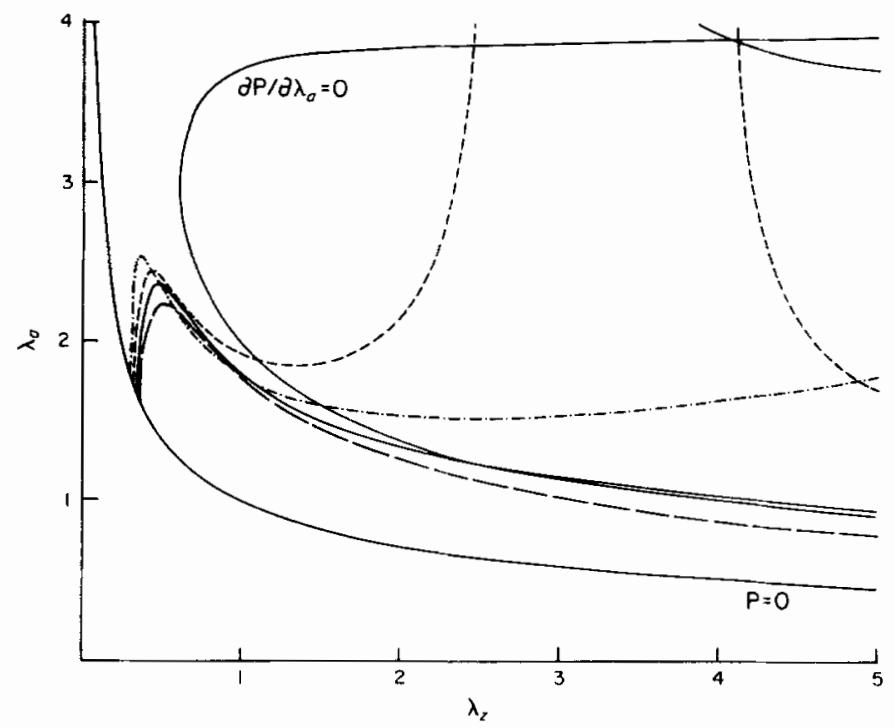

(a)

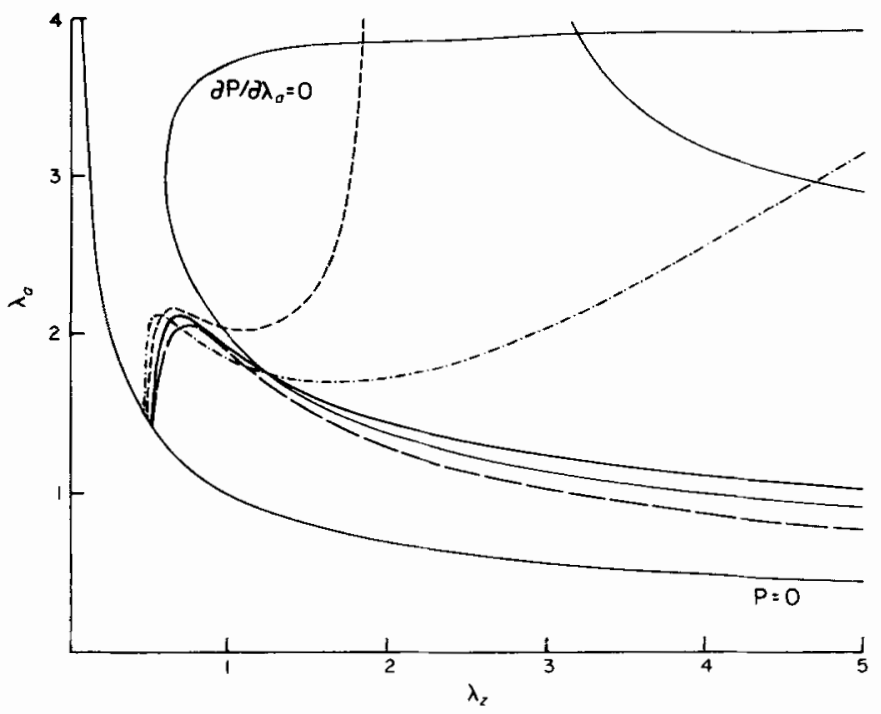

(b) 


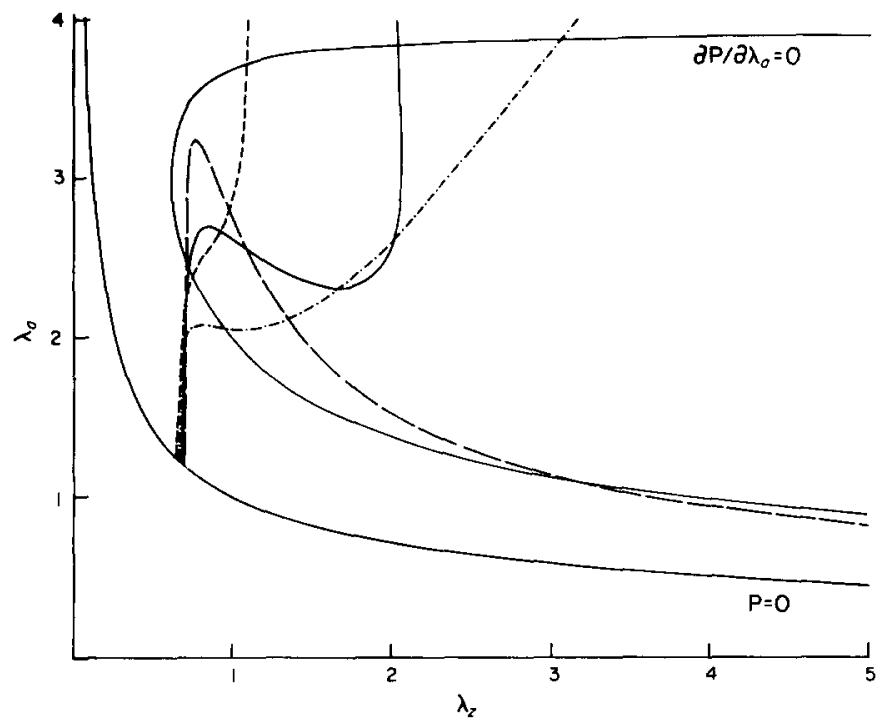

(c)

FIG. 3. Plot of the axisymmetric bifurcation curves in the $\left(\lambda_{2}, \lambda_{a}\right)$-plane for $P \geqslant 0$ and $A / B=0.85$ in respect

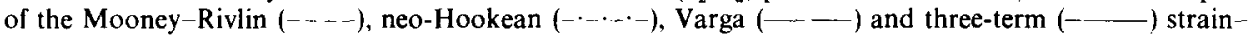
energy functions: (a) $L / B=20$, (b) $L / B=10$, (c) $L / B=5$. The curve of pressure turning-points (calculated for fixed $\lambda_{2}$ ) is included in (a), (b) and (c) for the three-term strain-energy function.

easily than shorter ones. However, it should be borne in mind that asymmetric (or bending) modes will have priority over axisymmetric modes for long tubes in compression (this is shown in Section 4.3).

For the neo-Hookean solid, WILKES (1955) considered a tube subject to end-thrust alone. In this special case, $\lambda^{2} \lambda_{z} \equiv 1$ so the underlying deformation is homogeneous and the equations can be solved in terms of modified Bessel functions. Wilkes (in different notation) plots $\lambda_{z}$ against $\alpha b \equiv n \pi B / L \lambda_{z}^{3 / 2}$ and the resulting curve provides a critical value of $\lambda_{2}$. Bifurcation into an axisymmetric mode in compression cannot occur for values of $\lambda_{z}$ greater than this critical value. Whether or not bifurcation occurs at the critical value depends on whether or not the value of $n B / L$ determined by the corresponding critical value of $\alpha b$ is an integral multiple of the ratio $B / L$ of the considered tube. The points of the curve $P=0$ in Fig. 5(a,b) corresponding to bifurcation in essence show the behaviour of $\lambda_{z}$ as a function of $n B / L$.

In Fig. $6(\mathrm{a}, \mathrm{b}, \mathrm{c})$, for $L / B=5,10,20$ respectively, the value of $\lambda_{a}$ at bifurcation is plotted against $A / B$ for a number of values of $\lambda_{z}$ to illustrate the dependence of bifurcation on the tube thickness.

For each value of $\lambda_{z}$ shown, there is clearly a "cut-off" value of the tube thickness beyond which axisymmetric bifurcations do not occur with increasing pressure. For long tubes, as illustrated in Fig. $6(\mathrm{~b}, \mathrm{c})$, the character of the results does not vary significantly with thickness except near the cut-off values. Indeed, for thicknesses up to, say, $60 \%$ of the critical value (for each $\lambda_{z}$ and $L / B$ ) the results are broadly similar to those found in (I) for a membrane and corresponding to the limit $A / B=1$ here. 


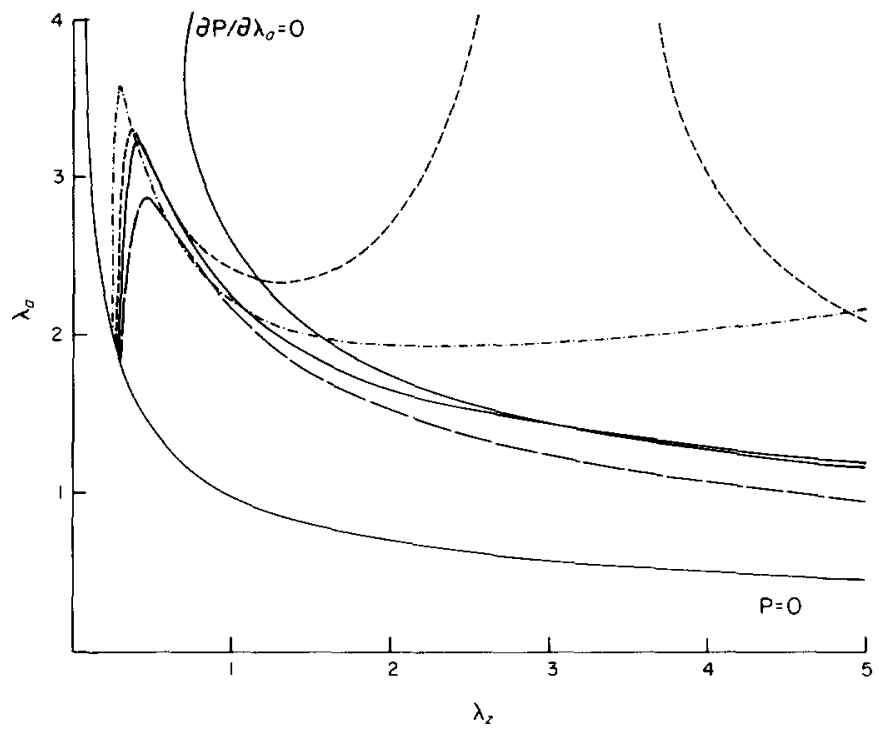

(a)

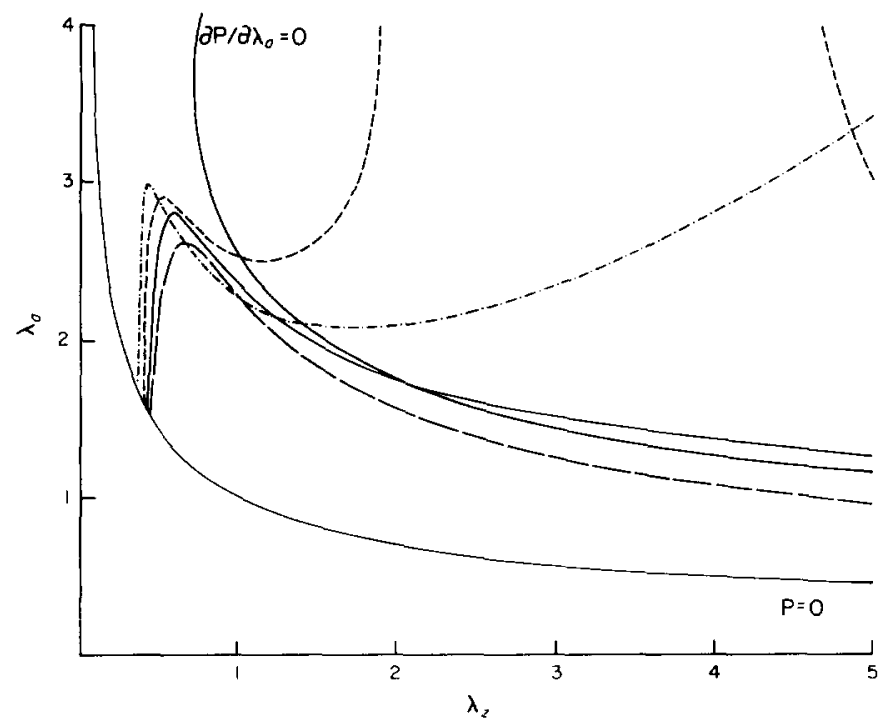

(b) 


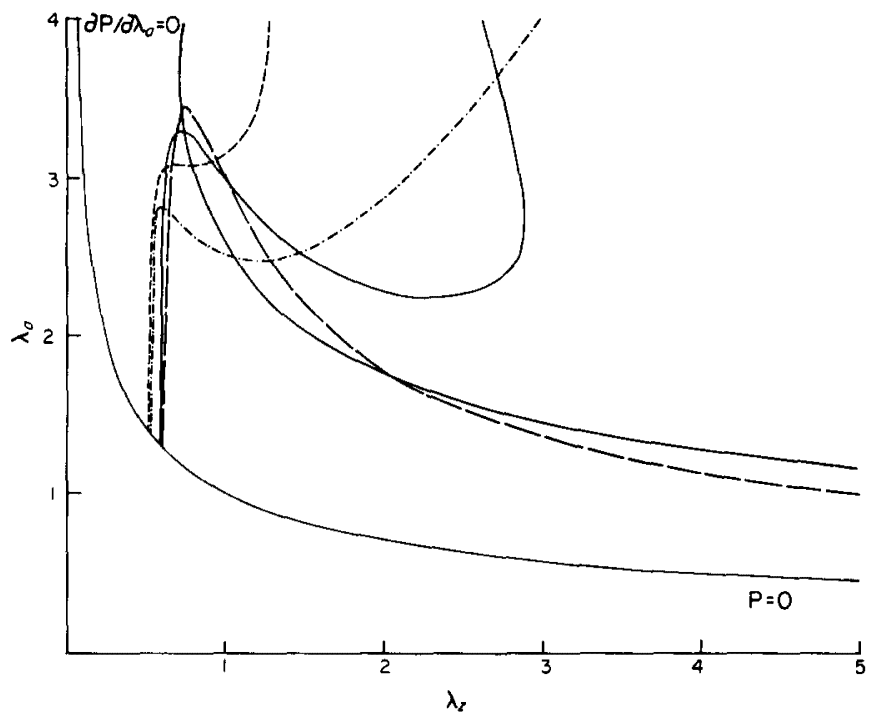

(c)

FIG. 4. Plot of the axisymmetric bifurcation curves in the $\left(\lambda_{z}, \lambda_{a}\right)$-plane for $P \geqslant 0$ and $A / B=0 \cdot 5$ in respect

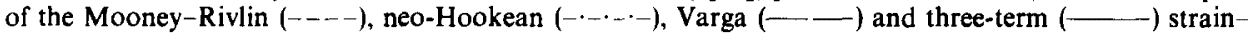
energy functions: (a) $L / B=20$, (b) $L / B=10$, (c) $L / B=5$. The curve of pressure turning-points (calculated for fixed $\lambda_{z}$ ) is included in (a), (b), (c) for the three-term strain-energy function.

Figure 6(a) shows that for short tubes the results depend more critically on the value of $A / B$. The relative positions of the bifurcation curves and the curves of pressure turning-points may be observed by comparing Fig. 6(a,b,c) with Fig. 2.

For a number of values of $\lambda_{z}$, the value of $\lambda_{a}$ at bifurcation is plotted against $B / L$ in Fig. 7, the left-hand limit corresponding to an infinitely-long tube. The ratio $A / B=0.85$ has been chosen here in view of the results in Fig. $6(\mathrm{a}, \mathrm{b}, \mathrm{c})$ which indicate that only for short tubes (that is, for $B / L$ larger than about $0 \cdot 15$ ) is there a significant dependence on $A / B$. Typical results for short tubes can be read off from Fig. 6(a).

The duality between the mode number $n$ and the ratio $B / L$ shows that for fixed $\lambda_{z}$ (or $B / L$ ) there is a value of $B / L$ (or $\lambda_{z}$ ) greater than which axisymmetric bifurcation cannot occur in any mode number.

\subsection{Asymmetric bifurcations}

When dependence on both $\theta$ and $z$ is retained, equations (23) become

$$
\begin{gathered}
\dot{p}_{\theta}=\left(r \cdot B_{3131}^{\prime}+\mathscr{B}_{3131}+\mathscr{B}_{1111}-\mathscr{B}_{1122}-\mathscr{B}_{2112}\right) u_{\theta} / r+\left(\mathscr{B}_{1111}-\mathscr{B}_{1122}-\mathscr{B}_{2112}\right) v_{\theta \theta} / r \\
+\mathscr{B}_{2121} r v_{z z}+\mathscr{B}_{3131} r v_{r r}+\left(\mathscr{B}_{1133}-\mathscr{B}_{1122}-\mathscr{B}_{2112}+\mathscr{B}_{3113}\right) u_{r \theta} \\
+\left(r \cdot \mathscr{B}_{3131}^{\prime}+\mathscr{B}_{3131}\right) v_{r}-\left(r \mathscr{B}_{3131}^{\prime}+\mathscr{B}_{3131}\right) v / r, \quad \\
\dot{p}_{z}=\mathscr{B}_{1212} w_{\theta \theta} / r^{2}+\left(r \mathscr{B}_{3223}^{\prime}+r p^{\prime}+\mathscr{B}_{3223}-\mathscr{B}_{1221}\right) u_{z} / r+\mathscr{B}_{3232} w_{r r} \\
+\left(\mathscr{B}_{2222}-\mathscr{B}_{1221}-\mathscr{B}_{1122}\right) w_{z z}+\left(\mathscr{B}_{2233}+\mathscr{B}_{3223}-\mathscr{B}_{1221}-\mathscr{B}_{1122}\right) u_{r z} \\
+\left(r \mathscr{B}_{3232}^{\prime}+\mathscr{B}_{3232}\right) w_{r} / r,
\end{gathered}
$$




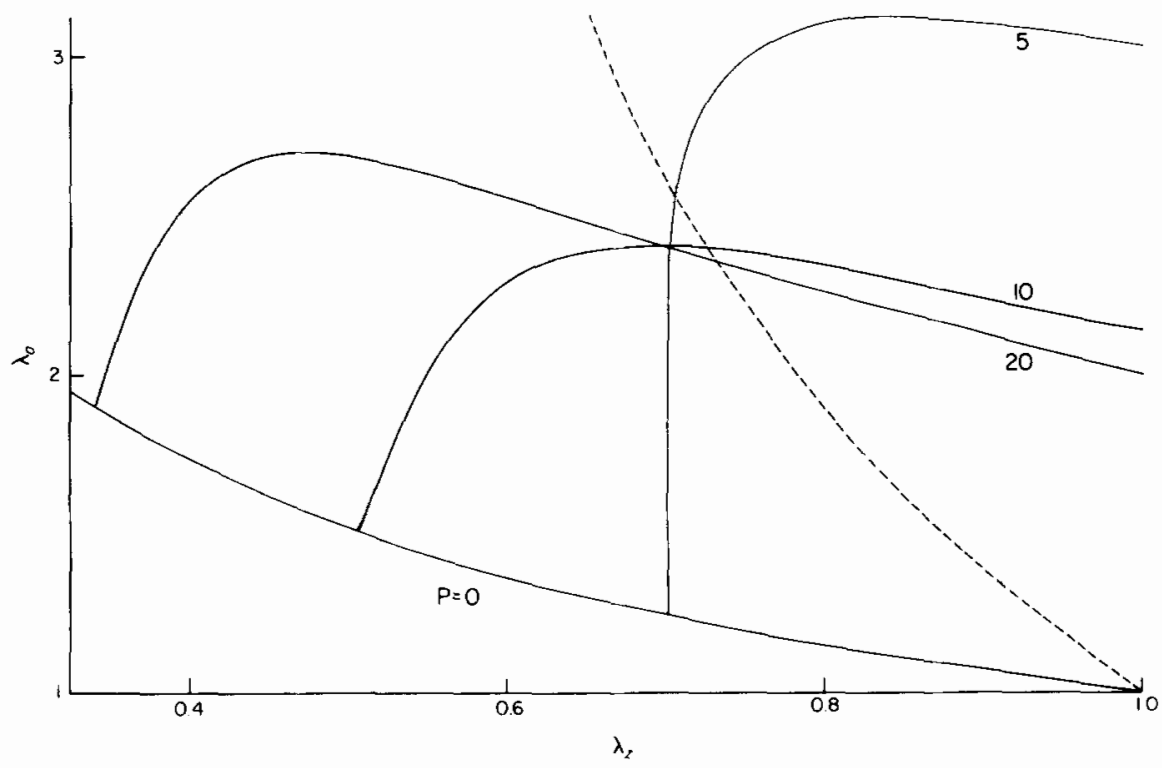

(a)

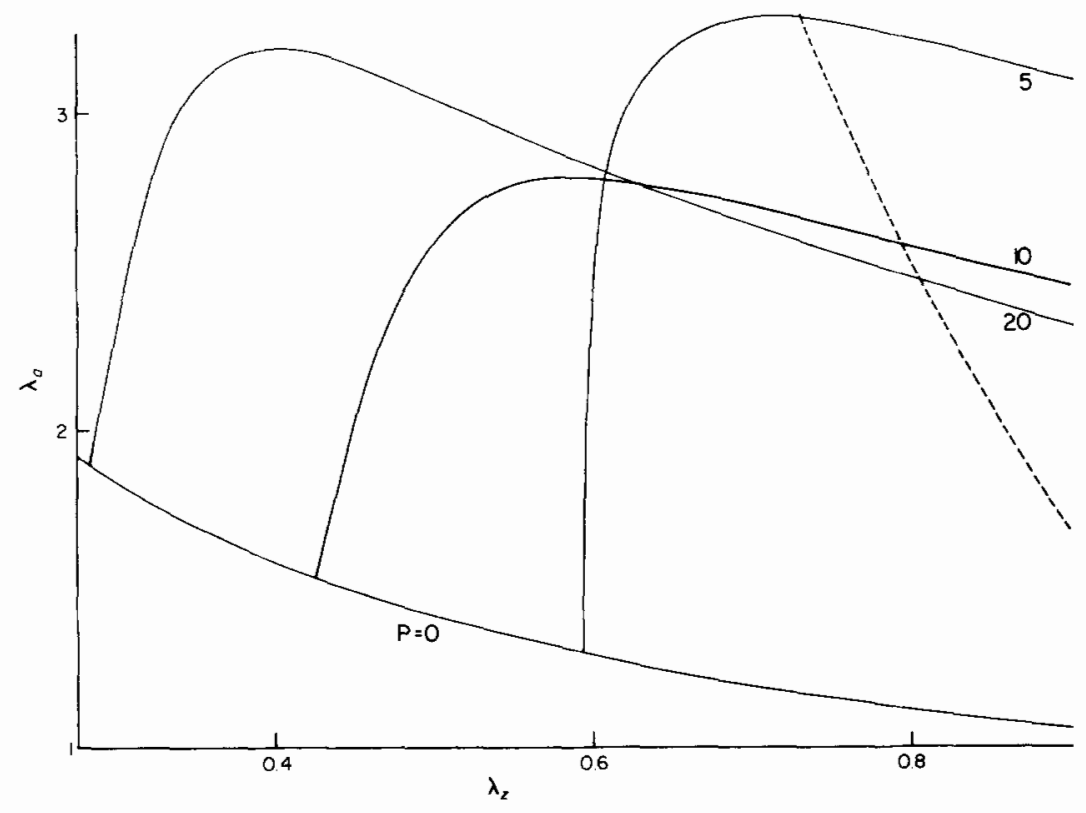

(b)

FIG. 5. Plot of the axisymmetric bifurcation curves for the region $\lambda_{z}<1$ and $P \geqslant 0$ of the $\left(\hat{\lambda}_{2}, \hat{\lambda}_{a}\right)$-plane with $L / B=5,10,20$ in respect of the three-term strain-energy function compared with the curve for $N=0$ $(---)$ : (a) $A / B=0.85$, (b) $A / B=0.5$. 


$$
\begin{aligned}
\dot{p}_{r}= & \mathscr{B}_{1313} u_{\theta \theta} / r^{2}+\left(r: B_{1133}^{\prime}-r \mathscr{B}_{2233}^{\prime}-\mathscr{B}_{1111}+\mathscr{B}_{1122}-\mathscr{B}_{1313}+\mathscr{B}_{3223}\right) v_{\theta} / r^{2} \\
& +\left(\mathscr{B}_{1331}+\mathscr{B}_{1133}-\mathscr{B}_{3223}-\mathscr{B}_{2233}\right) v_{r \theta} / r+\left(\mathscr{B}_{3333}-\mathscr{B}_{2233}-\mathscr{B}_{3223}\right) u_{r r} \\
& +\mathscr{B}_{2323} u_{z z}+\left(r \mathscr{B}_{3333}^{\prime}+r p^{\prime}-r \mathscr{B}_{2233}^{\prime}+\mathscr{B}_{3333}-2 \mathscr{B}_{2233}+\mathscr{B}_{1122}-\mathscr{B}_{3223}\right) u_{r} / r \\
& +\left(r \mathscr{B}_{1133}^{\prime}-r \mathscr{B}_{2233}^{\prime}-\mathscr{B}_{1111}+\mathscr{B}_{1122}+\mathscr{B}_{3223}\right) u / r^{2} .
\end{aligned}
$$

The incompressibility condition (26) is also required.

The equations are solved by setting

$$
\left.\begin{array}{rl}
u & =f(r) \cos m \theta \sin \alpha z, \\
v & =g(r) \sin m \theta \sin \alpha z, \\
w & =h(r) \cos m \theta \cos \alpha z, \\
\dot{p} & =k(r) \cos m \theta \sin \alpha z,
\end{array}\right\}
$$

the $z$-dependence selected, as in (59), in order to satisfy end-conditions which again lead to values of $\alpha$ given by (60). In order that the solutions be single-valued we take $m=0,1,2, \ldots$.

The expressions (65) are substituted in (62)-(64) and $h(r)$ is eliminated by means of (26). The resulting coupled equations for $f(r), g(r)$ and $k(r)$ are then put in the form

$$
\frac{d y_{i}}{d r}=F_{i}(\mathbf{y}, r) \quad(i=1, \ldots, 6),
$$

where

$$
\mathbf{y}=\left(f, f^{\prime}, f^{\prime \prime}, g, g^{\prime}, k\right),
$$

suitably non-dimensionalized.

The corresponding boundary conditions, after some algebra, can be put in the form

$$
\left.\begin{array}{l}
r y_{5}-y_{4}-m y_{1}=0, \\
r^{2} y_{3}+r y_{2}+\left(\alpha^{2} r^{2}+m^{2}-1\right) y_{1}=0, \\
\left(\mathscr{B}_{1133}-\mathscr{B}_{2233}\right)\left(y_{1}+m y_{4}\right)+\left(\mathscr{B}_{3333}-\mathscr{B}_{2233}+\sigma_{3}\right) r y_{2}-r y_{6}=0,
\end{array}\right\}
$$

each for $r=a, b$.

First, the initial-value problem defined by

$$
y_{i}(a)=\delta_{i k} \quad(i=1, \ldots, 6),
$$

where $\delta$ denotes the Kronecker delta, is examined for each of $k=1, \ldots, 6$.

Subject to (68) the equations (66) are solved using a fourth-order Runge-Kutta method. The six solutions thus generated are denoted by $\mathbf{y}^{k}(k=1, \ldots, 6)$ and the solution of the problem with boundary conditions (67) is expressible in the form

$$
\mathbf{y}=\sum_{k=1}^{6} c_{k} \mathbf{y}^{k}
$$

where the $c_{k}$ are constants.

Bifurcation occurs if there exists a non-trivial set of constants $c_{k}$ such that (67) are satisfied for $r=a, b$. Substitution of (69) into (67) yields the bifurcation criterion, viz. the vanishing of the resulting $6 \times 6$ determinant of coefficients of $c_{k}$. 


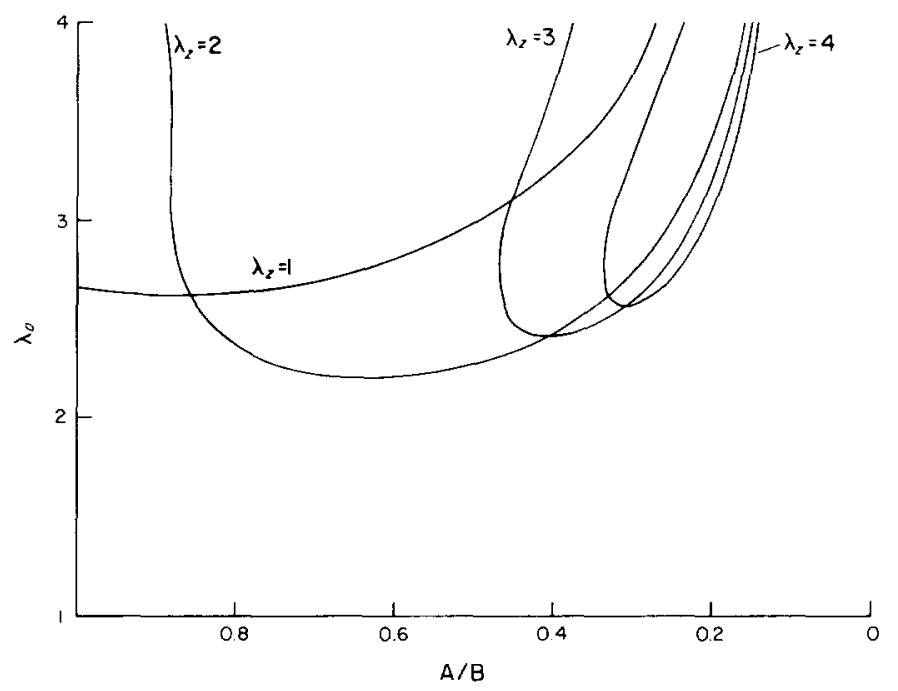

(a)

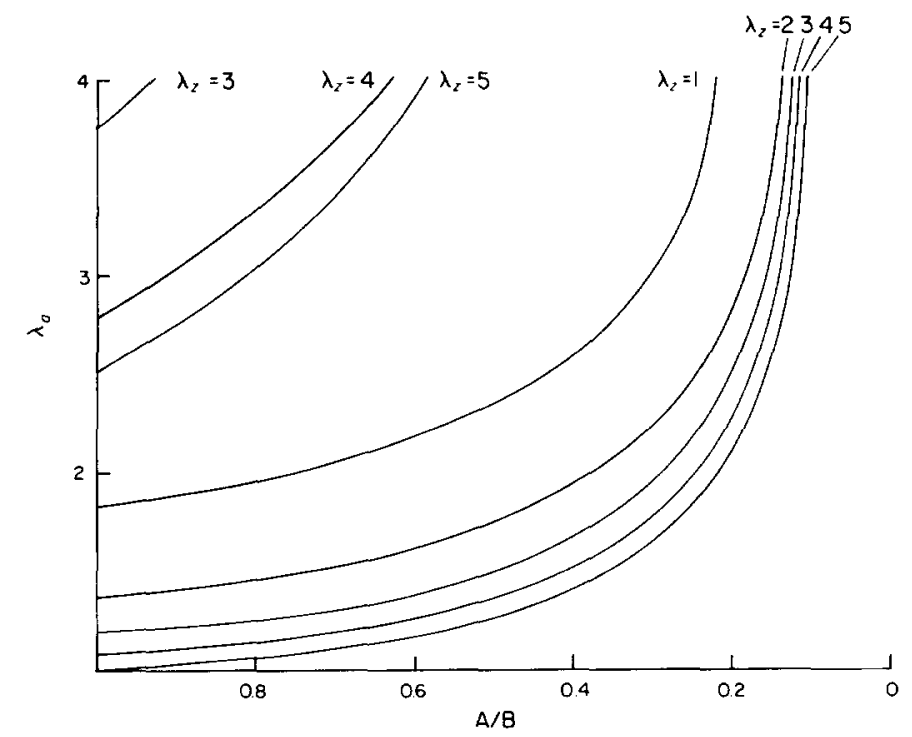

(b) 


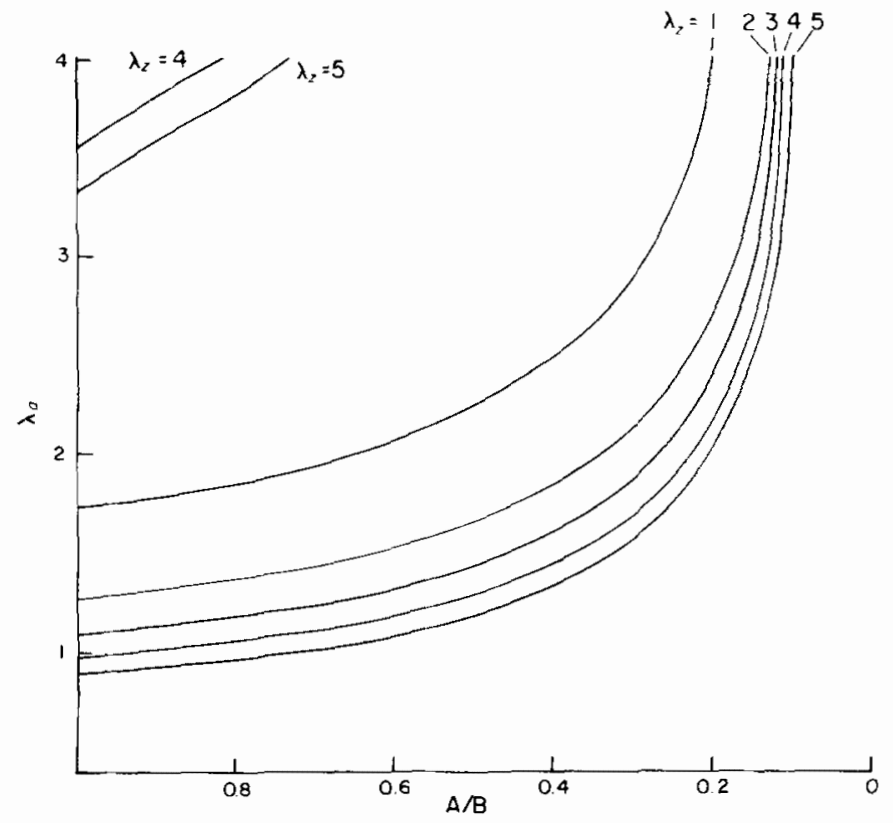

(c)

FIG. 6. Plot of the value of $\lambda_{a}$ at bifurcation against $A / B$ for $\lambda_{z}=1,2,3,4,5$ in respect of the three-term strain-energy function: (a) $L / B=5$, (b) $L / B=10$, (c) $L / B=20$.

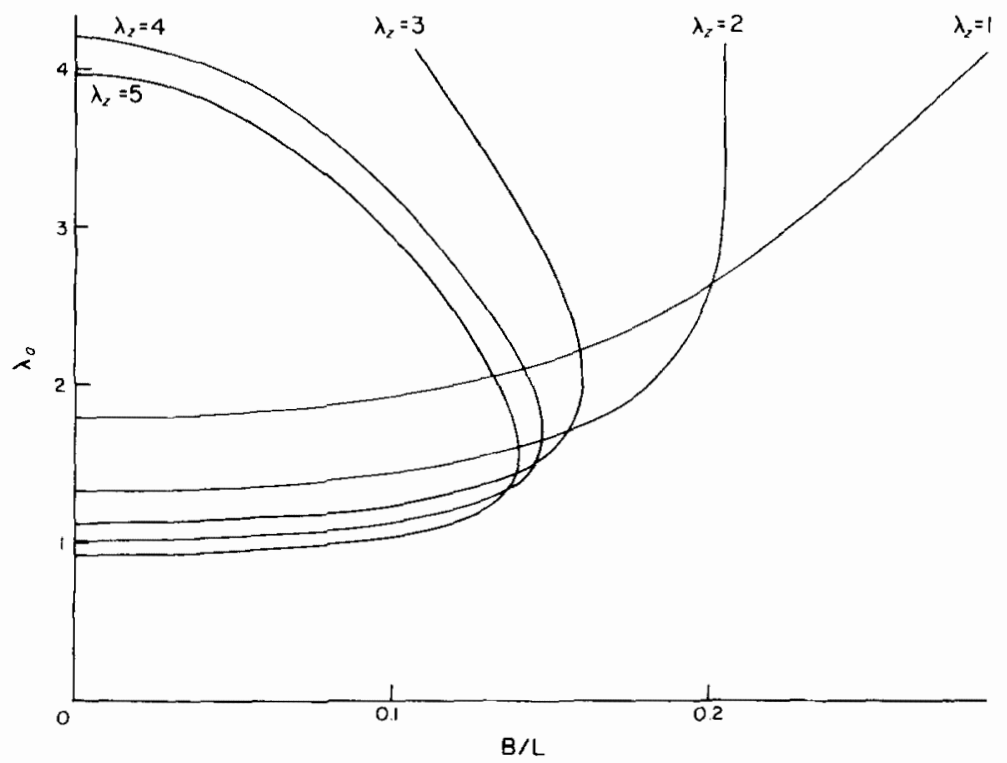

Fig. 7. Plot of the value of $\lambda_{a}$ at bifurcation against $B / L$ for $\lambda_{z}=1,2,3,4,5$ with $A / B=0.85$ in respect of the three-term strain-energy function. 


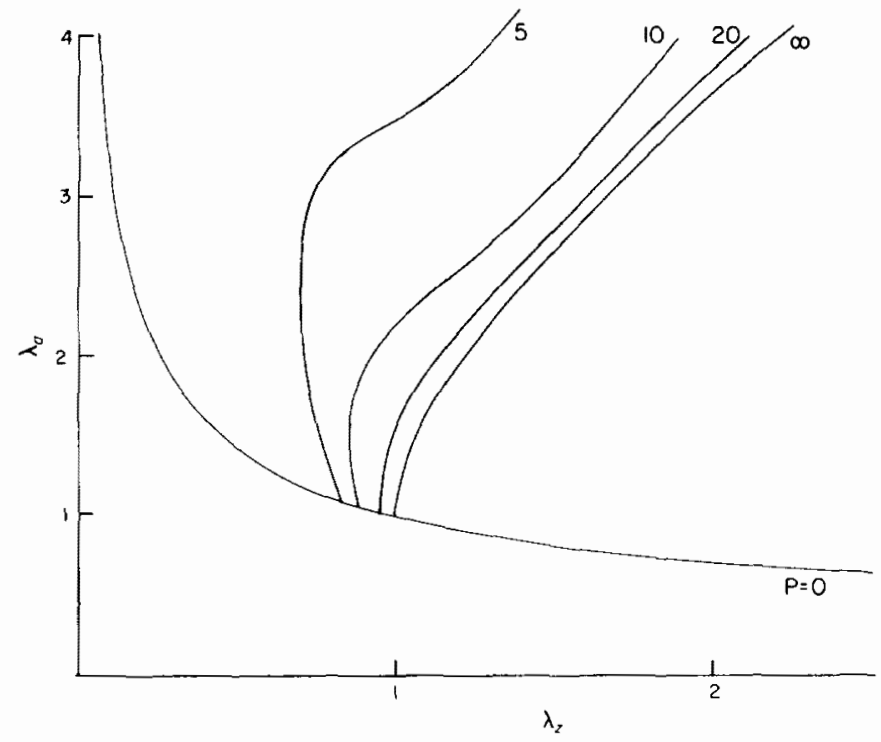

(a)

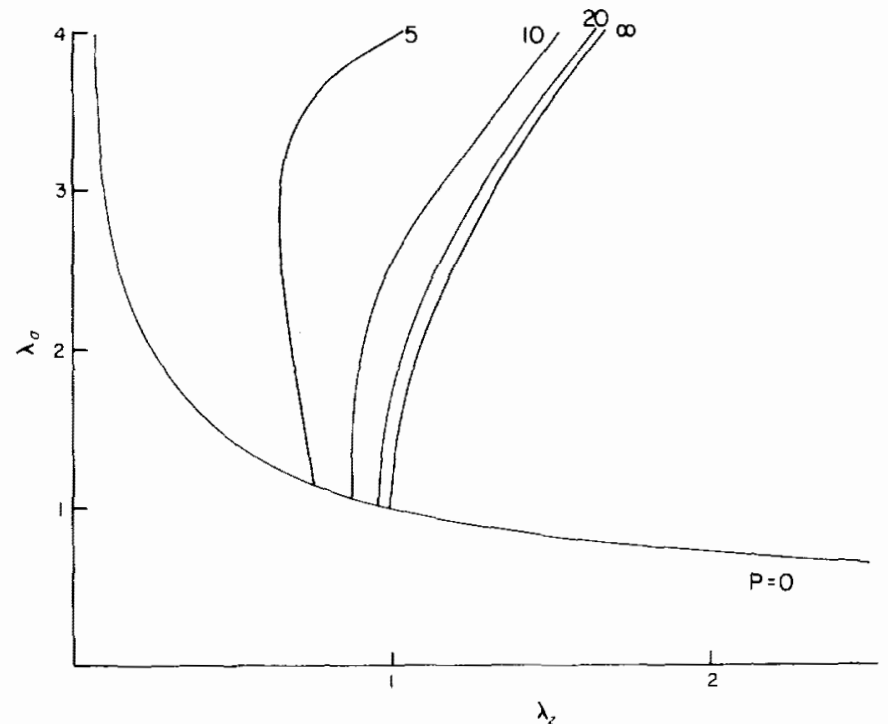

(b)

FIG. 8. Plot of the asymmetric bifurcation curves in the $\left(\lambda_{z}, \lambda_{a}\right)$-plane for $P \geqslant 0$ and $L / B=5,10,20, \infty$ in respect of the three-term strain-energy function: (a) $A / B=0.85$, (b) $A / B=0.5$. 
It is an easy matter to find a value of $a$ (if one exists in the range under consideration), and hence $b$ from $b^{2}=\lambda_{z}^{-1}\left(B^{2}-A^{2}\right)+a^{2}$, to satisfy the bifurcation criterion as accurately as required.

This numerical method has in essence been used by ERTEPINAR (1978) to investigate bifurcation of thick-walled tubes of neo-Hookean material subjected to finite twist combined with external pressure.

The above method was the basis for our calculations (corresponding to $m=0$ ) described in Section 4.2.

For $m=1$ and $\alpha$ given by (61), bifurcation curves are plotted in the $\left(\lambda_{z}, \lambda_{a}\right)$-plane in Fig. $8(\mathrm{a}, \mathrm{b})$ for $A / B=0.85,0.5$ respectively. The character of the results is essentially independent of $A / B$ and, in particular, there is no "cut-off" as there is in the case of axisymmetric bifurcations. This, of course, is to be expected since solid cylinders $(A / B=0)$ are susceptible to bending in compression. Bending can only occur when the dilatational part of the axial loading is compressive, that is when $N^{\prime}$, given by (19), is non-positive. In the membrane case of (I) this reduces to the integrand in (19) being non-positive.

In the region where $N^{\prime}>0$, asymmetric modes do not compete with axisymmetric modes, but where $N^{\prime} \leqslant 0$ whether an axisymmetric or asymmetric mode can occur first during inflation at fixed $\lambda_{z}$ depends on $\lambda_{z}$ and $L / B$, just as for a membrane (see (I)), and also on the ratio $A / B$. This can be seen by comparing Fig. $8(\mathrm{a}, \mathrm{b})$ with Fig. 5(a,b) respectively and also with the appropriate parts of Figs 3 and 4 respectively. Note, however, the differences in scale. We do not pursue a discussion of the comparison here since, for a membrane, this has been done in (I) and the details are broadly similar for thick-walled tubes provided $A / B$ is not too small.

For $m \geqslant 2$, and $\alpha$ again defined by (61), our calculations reveal no bifurcation curves in the region of the $\left(\lambda_{z}, \lambda_{a}\right)$-plane considered in respect of the strain-energy function (46).

\section{ACKNOWLEDGEMENT}

The work of D.M.H. was supported by a Research Grant from the U.K. Science Research Council.

\section{REFERENCES}

Chadwick, P. and HADDON, E. W.

Charrier, J.-M. and LI, Y. K.

ERTEPINAR, A.

ERTEPINAR, A.

HAUghton, D. M. and OGDEN, R. W.

$\dagger$ Designated (I) in the text.
1972

1977 Trans. Soc. Rheol. 21, 301.

1972 Ph.D. Dissertation. Theoretical and Experimental Studies on Shells of Arbitrary Wall-Thickness subjected to Internal and External Pressure. Drexel University, Philadelphia. (See Diss. Abstr. B33, (1), 760-B (1972).)

1978 Int. J. Solids Struct. 14, 715.

1978 J. Mech. Phys. Solids 26, 111.

1979 Ibid. 27, 179† 
HiLl, J. M.

NoWINSKI, J. L. and SHAHINPOOR, M. OGDEN, R. W.

PATterson, J. C.

SieRAKOWSKI, R. L.,

Sun, C. T. and

Ebcioglu, I. K.

Skala, D. P.

WANG, A. S. D. and ERTEPINAR, A.

WILKES, E. W.
1976 J. Elasticity 6, 113.

1969 Int. J. Non-Linear Mech. 4, 143.

1978 Math. Proc. Camb. phil. Soc. 83, 127.

1976 Int. J. Non-Linear Mech. 11, 385.

1975 Ibid. 10, 193.

1970 Rubber Chem. Technol. 43, 745.

1972 Int. J. Non-Linear Mech. 7, 539.

1955 Q.J. Mech. appl. Math. 8, 88. 